eCommons@AKU

June 2004

\title{
Operative treatment of bilateral hip dislocation in children with arthrogryposis multiplex congenita
}

S Asif

Aga Khan University

masood umer

Aga Khan University, masood.umer@aku.edu

Rashid Baig

Aga Khan University, rashid.baig@aku.edu

Follow this and additional works at: http://ecommons.aku.edu/pakistan_fhs_mc_surg_surg

Part of the Orthopedics Commons

\section{Recommended Citation}

Asif, S., umer, m., Baig, R. (2004). Operative treatment of bilateral hip dislocation in children with arthrogryposis multiplex congenita. J Orthop Surg, 12(1), 4-9.

Available at: http://ecommons.aku.edu/pakistan_fhs_mc_surg_surg/225 


\title{
O perative treatment of bilateral hip dislocation in children with arthrogryposis multiplex congenita
}

\author{
S Asif, M U mer, R Beg, M U mar \\ Division of O rthopedics, D epartment of Surgery, The Aga Khan University H ospital, Karachi, Pakistan
}

\begin{abstract}
Purpose. Arthrogryposis multiplex congenita (AMC) is a rare syndrome with multiple joint contractures. It is commonly believed that bilaterally dislocated hips associated with joint contractures should not be reduced, because movement is satisfactory, while open reduction leads to poor results. This report presents our experience with surgical management of bilateral dislocation of hips in children with AMC.

Methods. During the period 1990 to 2000, we performed open reduction on 8 hips of 4 children with AMC. The mean age at surgery was 23 months (range, 5-48 months). Open reduction and capsular plication without any bony procedure were performed in 4 hips (2 patients). De-rotation and varus osteotomy of the femur was performed in 4 hips, and Salter
\end{abstract}

osteotomy of the innominate bone in 2 hips. The average acetabular index was $44^{\circ}$, and the mean centreedge angle was $-41^{\circ}$ preoperatively.

Results. The average follow-up period was 4 years (range, 2-9 years). The average acetabular index and centre-edge angle were $19^{\circ}$ and $18^{\circ}$, respectively at the time of last follow-up. All children could walk without support. One child required re-opening for redislocation of hip joint. The clinical results were good in 6 hips and fair in 2 hips, according to Severin's and McKay's classifications.

Conclusion. Our experience shows that open reduction for bilateral dislocation of hips in children with AMC is a suitable option with generally good results. Surgery performed at earlier age gives the best functional outcome.

Key words: arthrogryposis, congenital; hip dislocation; osteotomy 


\section{INTRODUCTION}

Arthrogryposis multiplex congenita (AMC) is a syndrome complex characterised by several features including multiple joint involvement with severe contractures, dislocations, lack of normal skin creases, and varying degree of fibrosis of the affected muscles. ${ }^{1-3}$ Otto $^{4}$ first described this condition in 1841, but Stern ${ }^{5}$ was the first to coin the term "arthrogryposis multiplex congenita" to describe 4 cases reported in 1923. Hall $^{6}$ classified AMC into 3 broad categories according to the presence or absence of associated visceral and central nervous dysfunction. ${ }^{7}$ The incidence of AMC is rare, occurring in approximately $0.03 \%$ of newborn infants. ${ }^{2}$

Hip joint contracture with or without dislocation is reported in around $80 \%$ of patients with AMC. ${ }^{3,8}$ Isolated contractures are usually treated conservatively by manipulation and splinting, while operative intervention is required only occasionally. Closed reduction of the dislocated hip in children with AMC, however, has been relatively unsuccessful..$^{9-11}$ For unilateral dislocation, open reduction is recommended to prevent pelvic obliquity, imbalance in sitting, and eventual scoliosis. ${ }^{1,3,8}$ Some authors believe that the bilaterally dislocated hips should not be reduced, because the pelvis remains level and movement is satisfactory. ${ }^{1,9}$ On the contrary, others suggest that all dislocations should be reduced to restore normal hip mechanics, and to reduce the risk of future pain or stiffness. ${ }^{12,13}$ We have been performing routine open reduction on the bilaterally dislocated hips in children with AMC since 1990. We present our experience in dealing with such cases, and the functional and radiological results of these patients.

\section{PATIENTS AND METHODS}

In this retrospective study we reviewed all our patients with AMC who had undergone surgical treatment for bilateral dislocation of the hips (4 children with 8 hips) at the Division of Orthopedics, The Aga Khan University Hospital, Karachi, from 1990 to 2000. The objectives of this study were to report the outcome of surgical management of bilateral dislocation of the hips in patients with AMC, and to analyse the complications of treatment. We included in our study only those patients who fulfilled the following criteria:

(1) Joint contracture at birth in at least 2 different parts of the body;

(2) Bilateral dislocation of the hips;

(3) Generalised muscle wasting; and

(4) Absence of normal skin creases.
Four children, with 8 dislocated hips, met our criteria. All of them had bilateral talipes equinovarus. One child had bilateral knee dislocation, and one had hand deformities (finger contractures and clasped thumb). No patient had any central nervous system or visceral abnormality. With only limb involvement, these patients were classified as type I according to Hall. ${ }^{6,7}$ Three patients were females and one was male. The average age at the time of surgery was 23 months (range, 5-48 months).

In the child with bilateral knee and hip dislocations, the knees were operated on before the hips. This sequencing facilitated the performance and positioning during hip surgery and the application of hip spica cast. The feet of all children except one were corrected outside our institution before presentation. A child who was 6 months old at the time of presentation had the hips operated on first. The feet were corrected at the age of one year, once the child was out of the spica cast.

All the preoperative clinical assessments were recorded. The mean fixed flexion contracture at the hip joint was $20^{\circ}$, mean maximum flexion at hip $70^{\circ}$, mean adduction $25^{\circ}$, mean abduction $15^{\circ}$, mean internal rotation $35^{\circ}$, and mean external rotation $35^{\circ}$. The telescoping test yielded positive results in all cases. We assessed radiologically the patient's hips preoperatively by the Tonnis grading system, ${ }^{14,15}$ measurement of acetabular index, and centre-edge angle. Preoperatively 4 of the hips were in grade IV, one was in grade III, and 3 were in grade II according to the Tonnis grading system. The average acetabular index before operation was $44^{\circ}$, and the average centre-edge angle was $-41^{\circ}$.

All patients were managed by two surgeons. The hips were approached through the iliofemoral incision. All the tight muscles, sartorius, iliopsoas, and the rectus femoris were incised. The capsule of the hip joint was opened in a " $\mathrm{T}$ " shape. The acetabulum was cleared of any fibro fatty tissue and the femoral head reduced. Then by performing a full range of passive movements in all directions, we assessed stability of the hip joint. All unstable hips were immediately stabilised by an innominate osteotomy. A femoral shortening was done in those hips which seemed tight after reduction, or when reduction was difficult. Meticulous capsular plication was performed with strong sutures at the end of all cases. Four hips were stable after open reduction and capsular plication. In 2 cases of hip reduction, stability was achieved after femoral shortening and de-rotational varus osteotomy. In 2 hips, Salter innominate osteotomy was needed to cover the femoral head after doing the femoral shortening and de-rotational varus osteotomy. A hip spica was given postoperatively for 3 months. 


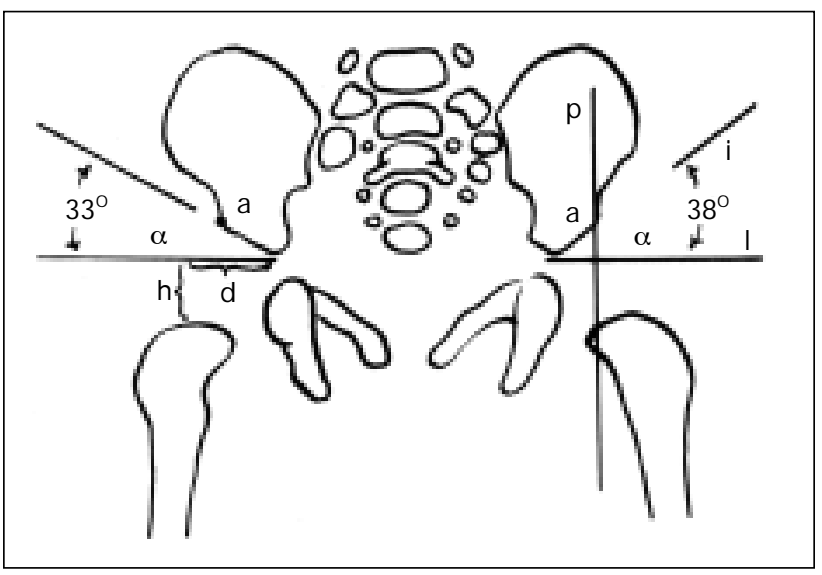

Figure 1 M easurement of acetabular index. It is increased on the left side.

Patients were then allowed gradual ambulation and range-of-motion exercises with advice for night splinting for another 3 months. The average followup period was 4 years (range, 2-9 years), and none of the patients were lost to follow-up at the time of writing this report. All the hip joints were assessed clinically and radiologically at each follow-up visit; Tonnis grading system ${ }^{14,15}$ and Severin's classification ${ }^{14,16}$ were used for radiological assessment. We also measured the acetabular index (Fig. 1) and centre-edge angle (Fig. 2) during follow-up. Clinical assessment of the hips was done according to McKay's ${ }^{14,17}$ score.

\section{RESULTS}

At the time of the final follow-up, 7 of our hips were in grade I, and one was in grade II according to the Tonnis grading system, whereas the average acetabular index was $25^{\circ}$ and average centre-edge angle was $18^{\circ}$.

Five hips were in class Ia, and one each in Ib, IIa and, IIIlb according to Severin's classification. Six hips were good and 2 were fair according to the McKay's score. The mean maximum flexion at the hip joint was $100^{\circ}$, mean adduction $30^{\circ}$, mean abduction $30^{\circ}$, mean internal rotation $70^{\circ}$, mean external rotation $60^{\circ}$, and the mean fixed flexion contracture $5^{\circ}$. All patients were ambulant without any support at the time of the final follow-up.

There was complication in 2 hips. In one hip in which we performed open reduction and femoral derotation varus osteotomy, dislocation occurred in the first postoperative week. This required revision of open reduction and plication of the capsule. The patient did well after this revision and no dislocation occurred

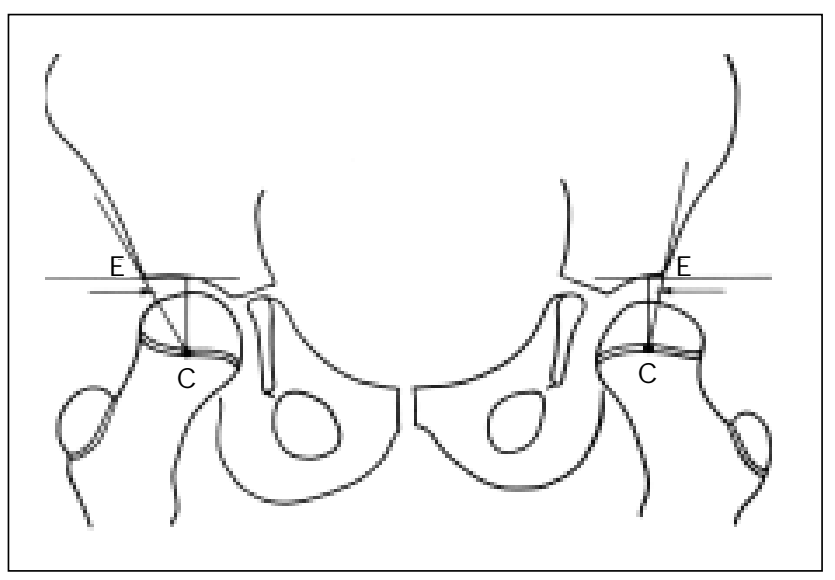

Figure 2 Measurement of centre-edge angle. The left hip is dysplastic and the centre-edge angle is decreased on that side.

again. The other hip, where we performed open reduction and plication of the capsule, had residual subluxation of the joint at 6 months. This hip was treated by bracing, and produced no further problems.

\section{DISCUSSION}

Dislocated hip in AMC is usually defined as a teratologic dislocation that occurs early during foetal development. A teratologic dislocation is much more rigid than the simple idiopathic congenital dislocation, because the intrauterine hip remodelling process has been disturbed at an earlier time in gestation. ${ }^{1,9,11}$ The basic patho-mechanism of AMC appears to be the lack of foetal movements, and foetal akinesia. Hall ${ }^{6,7}$ has designated AMC as amyodysplasia because of the apparent absence or hypoplasia of the muscles. However, during embryonic stage the muscles are actually formed normally, but during the foetal period they are replaced by fibrous and fatty tissues.

An incidence rate of hip joint contracture of $80 \%$, with or without dislocation, has been reported in patients with AMC. ${ }^{3,8}$ It is generally believed that unreduced unilateral dislocation will later give rise to pelvic obliquity and ultimately scoliosis, hence open reduction of unilateral dislocation in AMC is recommended. ${ }^{1,8,12}$ The management of bilateral dislocation, however, has been controversial. The high rate of complications such as redislocation, stiffness, ${ }^{10}$ and avascular necrosis ${ }^{3}$ has led to the recommendation that bilateral dislocation should be left unreduced. ${ }^{1,8}$

St Clair and Zimbler ${ }^{12}$ divided the bilateral dislocation of hips into 2 subgroups. The first includes 


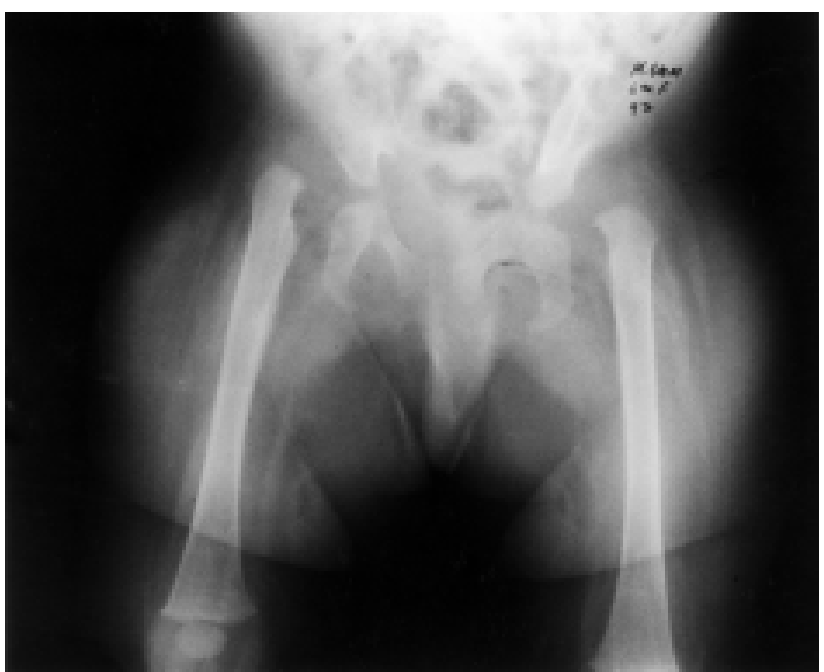

(a)

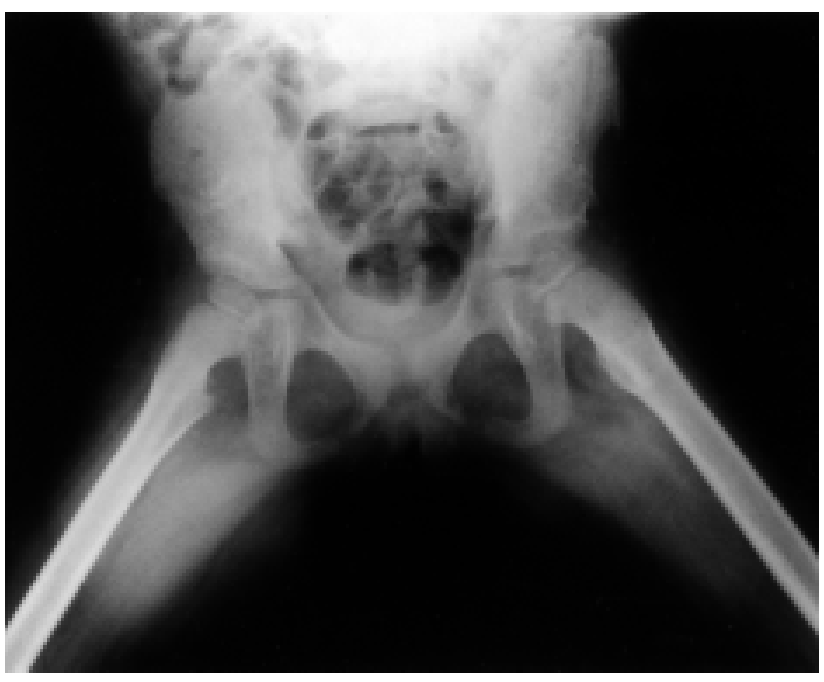

(c)

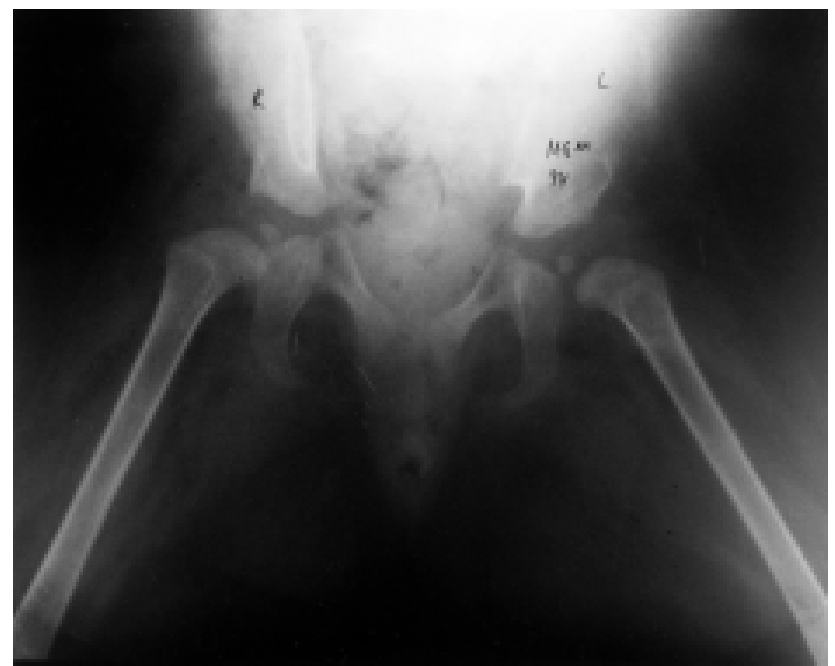

(b)

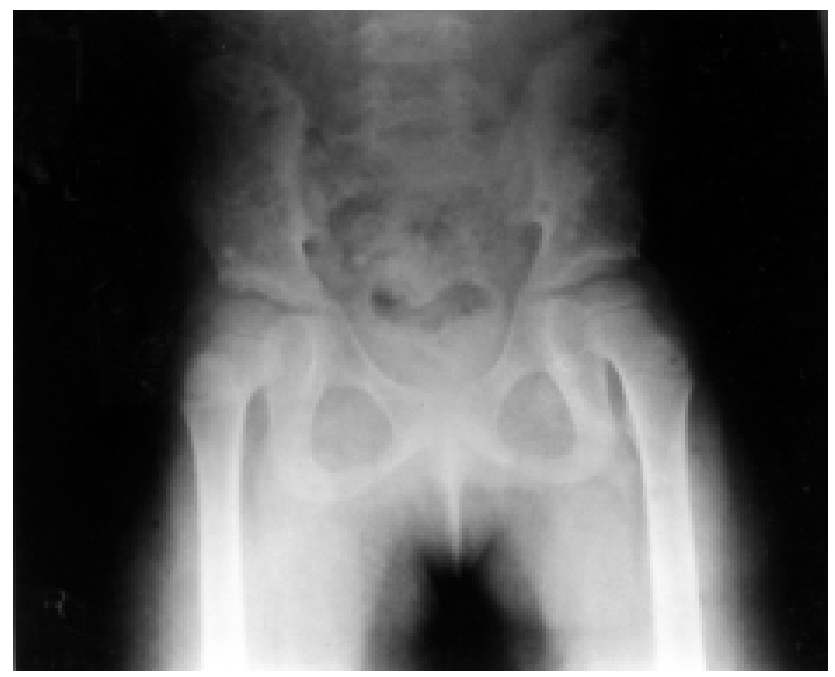

(d)

Figure 3 (a) A 6-month-old female child with AMC and bilaterally dislocated hips. Bilateral open reduction and capsular plication were performed. The hip joints were well reduced as shown in the radiographs taken at the age of (b) 18 months, (c) 5 years, and (d) 9 years.

bilateral dislocation of hips with marked limitation of motion and severe involvement of the upper extremity. Because all these patients have the most severe form of this disorder, and their ambulatory potential is minimal, it has been recommended that open reduction should not be attempted in this group. The second subgroup consists of those with bilateral dislocation, less severe involvement of the upper extremity, and greater range of motion at the hips. They recommended open reduction of such hips. Because bilateral dislocation is not consistent with a good gait, they also recommended that open reduction be performed during the first year of life.
Lloyd et al. ${ }^{10}$ operated on 6 patients with bilateral hip dislocation, and all of them ended up with stiff joints. Staheli et al. ${ }^{13}$ operated on 3 patients with bilateral dislocation in AMC. He did a combination of open reduction, femoral varus de-rotational osteotomy and/or pelvic osteotomy, and showed good results. Akazawa et al. ${ }^{3}$ operated on 5 patients with bilateral dislocation in AMC in which complete circumferential capsulotomy was done; these patients had a high incidence of avascular necrosis.

In our series, we operated on 8 hips of 4 patients. Open reduction and plication of capsule (Fig. 3) was performed in 4 hips ( 2 patients). These 2 patients 
presented to us early, at the age of 6 months and 12 months, respectively. Both patients had low dislocation of the hips, and we were able to reduce and maintain the femoral head with plication of the capsule without bony procedure. On long-term follow-up, both patients were ambulant without support and had a reasonably good range of motion of their hip joints in

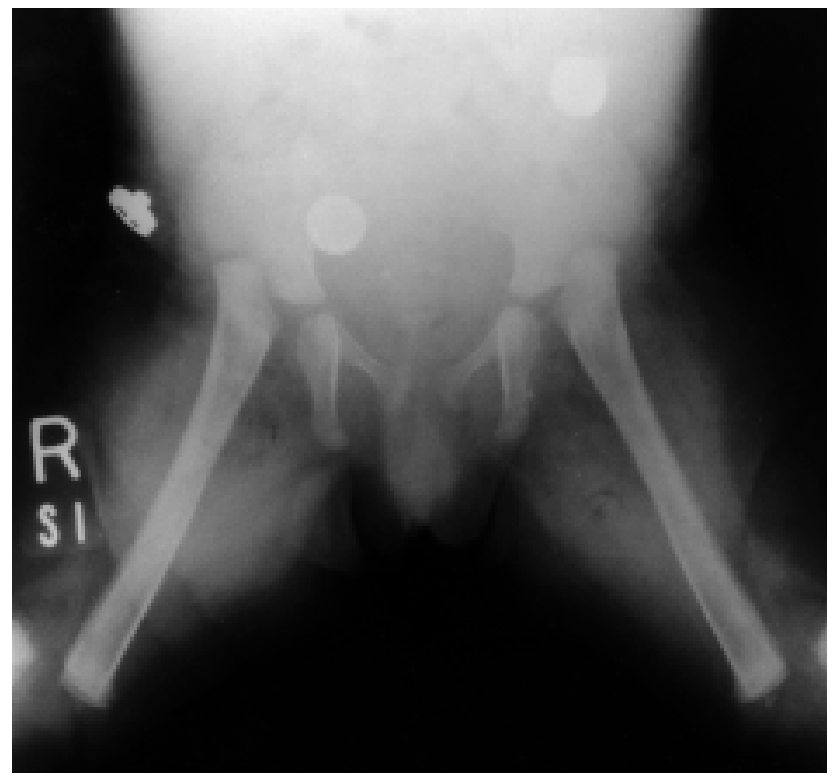

(a)

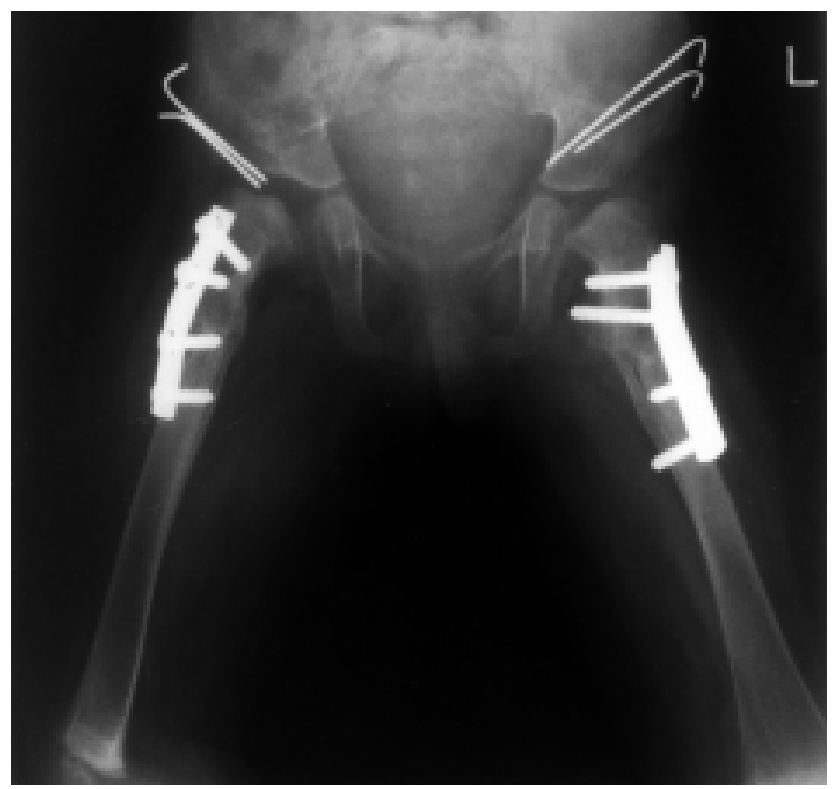

(b) all directions i.e. hip flexion of more than $110^{\circ}$, and no fixed flexion contracture. Both patients had greater than $70^{\circ}$ of internal and external rotations, and about $40^{\circ}$ of abduction on either side. The remaining 4 hips required additional bony procedures. In all these 4 hips, femoral shortening and varus de-rotational osteotomy were done to improve reduction and decrease the pressure on the femoral head. In 2 of these hips we also needed Salter innominate osteotomy to provide further stability to the hip joint (Fig. 4).

There were 2 complications in our series. In one hip, in which we did open reduction, revision of the capsular plication had to be done, because this hip could not be reduced by closed means after an acute postoperative dislocation. In the other hip, in which we did open reduction and plication of the capsule, residual subluxation was treated and improved with a brace. All other hips had a good range of motion, and all our patients were ambulant without crutches or canes at the time of writing this report.

The absence of comparative non-surgical controls and the small size of this series make it difficult to draw any statistical conclusion. Our results are, however, quite promising in both clinical and radiological terms. Left untreated, these hips would have progressive

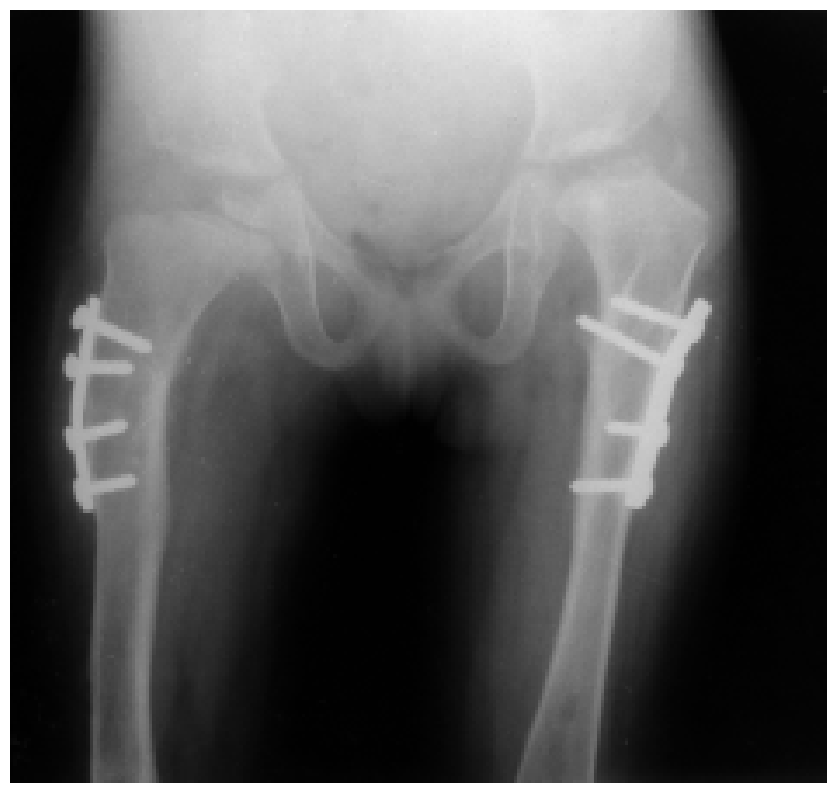

(c)

Figure 4 Radiographs showing (a) an 18-month-old child with bilateral teratologic dislocation of the hip joints secondary to AMC. Bilateral open reduction, femoral shortening and de-rotational osteotomy, and Salter's innominate osteotomy was thus performed. Follow-up radiographs at (b) 3 months and (c) 4 years after surgery showing good healing of the osteotomy and reduction of both joints. 
disability both in terms of restriction of activity and development of pain. Both of these disabilities were successfully eliminated by the surgical option. These children became much more functional after the surgery, and their parents were quite satisfied with the final outcome of their children.

\section{REFEREN CES}

1. Benson MK, Fixen JA, Malcom F, M acnicol M, Parsch L, editors. Children's orthopaedics and fractures. 1st ed. Edinburgh: Churchill Livingstone; 1994.

2. Thompson GH, Bilenker RM. Comprehensive management of arthrogryposis multiplex congenita. Clin O rthop 1985;194: 6-14.

3. A kazawa $\mathrm{H}, \mathrm{O}$ da K, Mitani S, Yoshitaka T, Asaumi K, Inoue $\mathrm{H}$. Surgical management of hip dislocation in children with arthrogryposis multiplex congenita. J Bone Joint Surg Br 1998;80:636-40.

4. O tto AW. A human monster with inwardly curved extremities. 1841. Clin O rthop 1985;194:4-5.

5. Stern W G. Arthrogryposis multiplex congenita. JAM A 1923;81:1507-10.

6. Hall JG. Genetic aspects of arthrogryposis. Clin O rthop 1985;194:44-53.

7. Tachdjian MO. Pediatric orthopedics. 2nd ed. Philadelphia: Saunders; 1990.

8. Huurman WW, Jacobsen ST. The hip in arthrogryposis multiplex congenita. Clin O rthop 1985;194:81-6.

9. D rummond DS, Siller TN, Cruess RC. Management of arthrogryposis multiplex congenita. AAOS Instructional Course Lectures. St Louis: CV M osby; 1974:79-95.

10. Lloyd Robert GC, Leltim AW. Arthrogryposis multiplex congenita. J Bone Joint Surg Br 1970;52:494.

11. Sarwark JF, MacEwen GD, Scott CI Jr. Amyoplasia (a common form of arthrogryposis). J Bone Joint Surg Am 1990;72: 465-9.

12. St Clair HS, Zimbler S. A plan of management and treatment results in the arthrogrypotic hip. Clin Orthop 1985;74-80.

13. Staheli LT, Chew DE, Elliott JS, M osca VS. M anagement of hip dislocation in children with arthrogryposis. J Pediatr O rthop $1987 ; 7: 681-5$.

14. OIney B, Latz K, Asher M. Treatment of hip dysplasia in older children with a combined one-stage procedure. Clin O rthop 1998;215-23.

15. Tonnis D. Congenital dysplasia and dislocation of the hip in children and adults. Berlin: Springer Verlag; 1987.

16. Severin E. Contribution to the knowledge of congenital dislocation of the hip: Late results of closed reduction and arthrographic studies of recent cases. Acta Chir Scand 1941;84(Suppl 63):1S-142S.

17. McKay DW. A comparison of the innominate and the pericapsular osteotomy in the treatment of congenital dislocation of the hip. Clin O rthop 1974;98:124-32. 